\title{
Desempenho de famílias de cana-de-açúcar em diferentes fases no melhoramento genético via REML/BLUP
}

\section{Performance of sugarcane families at different stages in the genetic improvement by REML/BLUP}

\author{
Ana Selena Fernández Lucius ${ }^{1}$; Ricardo Augusto de Oliveira ${ }^{2 *}$; \\ Edelclaiton Daros²; José Luis Camargo Zambon²; \\ João Carlos Bespalhok Filho²; Mario Alvaro Aloisio Verissimo ${ }^{3}$
}

\section{Resumo}

O objetivo deste trabalho foi selecionar as melhores famílias de cana-de-açúcar para aumento de produtividade $(\mathrm{TCH})$ na fase $\mathrm{T} 1$ e acompanhar seu desempenho nas fases $\mathrm{T} 2$ e $\mathrm{T} 3$ do programa de melhoramento genético da cana-de-açúcar (PMGCA/UFPR/RIDESA). Os experimentos das fases T1 e T2 foram realizados na estação experimental de Paranavaí (PR) e a fase T3 foi instalada em três locais da região noroeste do estado do Paraná. Na primeira fase (T1) foram avaliadas 40 famílias de irmãos-completos oriundos de cruzamentos bi-parentais e foram correlacionados com as famílias selecionadas na etapa seguinte de seleção (T2), comparando o número de genótipos indicado pelo BLUPIS e o número de clones que compuseram o T2, via seleção massal. Os dados foram analisados via metodologia REML/BLUP. Vinte e duas (22) famílias foram selecionadas para a fase T2 e sete famílias foram selecionadas para a fase T3, sendo resultados dos cruzamentos RB835486 x RB72454; RB855156 x SP70-1143; RB835054 x SP83-2847; RB835054 x SP80-3280; SP80-1816 x RB855156; SP80-3280 x RB855589; e SP80-3280 x RB835486. A seleção via procedimento BLUPIS permitiu indicar maior número de famílias potenciais e clones dentro destas famílias. Os genótipos com melhor desempenho nas fases seguintes foram originados das famílias com valores genotípicos positivos e produtividade acima da média experimental em T1.

Palavras-chave: Saccharum spp., correlação genética, herdabilidade, modelos mistos

\footnotetext{
Abstract

The objective of this work was to select the best sugarcane families to increase productivity (TCH) in T1 stage and monitor their performance in stages $\mathrm{T} 2$ and $\mathrm{T} 3$ of the sugarcane breeding program (PMGCA / UFPR / RIDESA). The experiments of stages $\mathrm{T} 1$ and $\mathrm{T} 2$ were performed at the experimental station of Paranavaí (PR) and T3 stage was installed in three locations in the northwestern of Paraná State. In the first phase (T1) 40 full-sib families from bi-parental crosses were evaluated and were correlated with selected families in the next stage of selection (T2), comparing the number of genotypes indicated by BLUPIS, and the number of clones that composed the T2 stage, by bulk selection. Data were analyzed by REML/BLUP methodology. In T1 stage, twenty and two (22) families were selected for T2 stage, and seven families were selected for T3 stage, being results of the crosses RB835486 x RB72454, SP70-1143 x RB855156, RB835054 x SP83-2847, SP80-3280 x RB835054, RB855156 x SP80-1816,

${ }^{1}$ Eng $^{\text {a }}$ Agr ${ }^{\mathrm{a}}$, M.e em Agronomia, Programa de Pós-graduação em Agronomia, Produção Vegetal, PGAPV, Universidade Federal do Paraná, UFPR, Curitiba, PR. E-mail: ana.selena@hotmail.com

${ }^{2}$ Eng $^{\text {os }}$ Agr $^{\text {os }}$, Profs. Drs. do Dept ${ }^{\text {o }}$ de Fitotecnia e Fitossanitarismo, UFPR, Curitiba, PR. E-mail: rico@ufpr.br; ededaros@ufpr.br; bespa@ufpr.br

3 Eng $^{\circ}$ Agr $^{\circ}$, Discente de Doutorado em Agronomia pelo PGAPV, UFPR, Curitiba, PR. E-mail: maaverissimo@gmail.com

* Autor para correspondência
} 
SP80-3280 x RB855589, and SP80-3280 x RB835486. The BLUPIS selection procedure allowed the indication of a greater number of potential families and clones within these families. The genotypes with improved performance in subsequent phases were derived from families with positive genotypic values and yield above experimental average in the T1 stage.

Key words: Saccharum spp., genetic correlation, heritability, mixed models

\section{Introdução}

A variabilidade genética disponível para seleção, em cana-de-açúcar, provém de cruzamentos realizados entre genitores de interesse que já apresentam um conjunto de genes relacionados a caracteres tidos como importantes componentes de produção (DUTRA FILHO et al., 2011).

No exemplo como ocorre no melhoramento de milho híbrido, é comum o uso de métodos para avaliar as linhagens com base em sua resposta nas combinações híbridas, com destaque para o topcross (ARNHOLD; VIANA; SILVA, 2009; BARRETO et al., 2012). No caso do melhoramento da canade-açúcar, os clones híbridos são resultados de cruzamentos entre genótipos com alta heterozigose (F1s), portanto não há a formação de linhagens homozigotas, e a combinação destas. Embora não se realizam topcrosses em cana-de-açúcar, o conhecimento do potencial do genótipo como genitor e das melhores combinações de genitores é de fundamental importância para obtenção de famílias superiores em cana-de-açúcar.

Neste sentido, a seleção de famílias vem se tornando uma boa estratégia, sobretudo quando estão envolvidos caracteres de baixa herdabilidade na seleção, como a produtividade de cana por hectare, TCH, (KIMBENG; COX, 2003; BARBOSA et al., 2004; OLIVEIRA et al., 2008; PEDROZO, et al., 2008). A seleção de famílias consiste em selecionar aquelas com elevados valores genotípicos, sendo descartadas famílias com valores genotípicos baixos (OLIVEIRA et al., 2011). Neste caso, a seleção de famílias com base em caracteres quantitativos de produção, poderá possibilitar a identificação de clones promissores com maior probabilidade de serem mais produtivos (OLIVEIRA et al., 2011).
A avaliação e seleção de progênies ou famílias é uma técnica comumente utilizada no melhoramento de diversas culturas, como por exemplo, milho (ARNHOLD; VIANA; SILVA, 2009), cana-deaçúcar (BARBOSA et al., 2005; OLIVEIRA et al., 2008), eucalipto (ROCHA et al., 2006), cenoura (SILVA; VIEIRA; NASCIMENTO, 2011), pequi (GIORDANI et al., 2012), entre outras. No entanto, em cana-de-açúcar, há escassez de informação a respeito do desempenho de famílias em diferentes fases do melhoramento genético, a fim de verificar a eficácia da seleção.

Segundo Stringer et al. (2011), são sólidas as evidências que mostram que uma combinação de seleção de famílias, seguida da seleção dentro da família é mais eficaz (maiores ganhos genéticos) e mais eficiente que a seleção individual sozinha. Ainda assim, a seleção de indivíduos dentro das famílias passa, necessariamente, pelo processo de avaliação visual, dada à grande dificuldade em obter dados em nível individual (RESENDE; BARBOSA, 2006; PEDROZO et al., 2008). Deste modo, Resende e Barbosa (2006) propuseram e Oliveira et al. (2011) confirmaram o uso da metodologia BLUP individual simulado (BLUPIS), que é um modelo matemático para indicar o número de indivíduos a ser selecionado visualmente por família, o número total de clones a ser avançado e o número de famílias a contribuir com indivíduos selecionados.

O objetivo deste trabalho foi avaliar famílias de irmãos-completos de cana-de-açúcar e a indicação de genótipos pelo método BLUPIS, seleção massal na primeira fase de seleção (T1) e avaliar o desempenho destas nas fases seguintes de seleção T2 e T3, através dos valores genotípicos preditos de cada indivíduo, da frequência de seleção de clones dentro das famílias e das correlações entre famílias selecionadas e a produtividade final. 


\section{Material e Métodos}

As fases de seleção (T1 e T2) foram instaladas na Estação Experimental de Paranavaí, localizada no município de Paranavaí, norte do Paraná (23 $34^{\prime}$ 02,75" de latitude Sul e 52 $32^{\circ}$ '53,87" de longitude Oeste, e altitude média de $450 \mathrm{~m})$. A adubação de base utilizada foi de $400 \mathrm{~kg} \mathrm{ha}^{-1}$, do formulado NPK 5:25:25, na proporção de $20 \mathrm{~kg} \mathrm{ha}^{-1}$ de $\mathrm{N}, 100 \mathrm{~kg}$ $\mathrm{ha}^{-1}$ de $\mathrm{K}_{2} \mathrm{O}$ e $100 \mathrm{~kg} \mathrm{ha}^{-1}$ de $\mathrm{P}_{2} \mathrm{O}_{5}$. A adubação em soca foi de $400 \mathrm{~kg} \mathrm{ha}^{-1}$, do formulado 20:0:20, sendo a proporção de $80 \mathrm{~kg} \mathrm{ha}^{-1}$ de $\mathrm{N}$ e $80 \mathrm{~kg} \mathrm{ha}^{-1}$ de $\mathrm{K}_{2} 0$ e com adubação de cobertura de $80 \mathrm{~kg} \mathrm{ha}^{-1}$ de N.

Para este estudo, foram utilizadas 40 famílias de irmãos-completos oriundas de cruzamentos biparentais entre 35 genitores. As hibridações foram realizadas na Estação de Cruzamento Serra do Ouro, Município de Murici, Estado de Alagoas, situada a $9^{\circ} 13^{\prime}$ de latitude Sul, 35 $50^{\circ}$ 'de longitude Oeste e altitude de 450 a 500 m. As progênies foram plantadas e mantidas em casa de vegetação, localizada no município de Paranavaí, Estado do Paraná. As plântulas foram transplantadas a campo em abril de 2001, formando a população base, ou T1, como é chamada a primeira fase de seleção nos programas de melhoramento da cana-de-açúcar.

\section{Fase T1}

$\mathrm{O}$ delineamento experimental na fase $\mathrm{T} 1$ foi em blocos incompletos, com os tratamentos compostos pelas 40 famílias de irmãos-completos e a parcela experimental constituída por 45 indivíduos por família, conforme descrito por Resende e Barbosa (2005), distribuídos em três linhas com 15 plantas por linha, espaçadas por 1,4 m entre linhas e 0,5 $\mathrm{m}$ entre plantas, e três repetições. $\mathrm{O}$ experimento foi colhido na cana-planta em abril de 2002, e a avaliação, realizada no ciclo de cana-soca em 2003.

A variável utilizada para análise das famílias foi tonelada de cana por hectare (TCH). A TCH foi estimada considerando a colheita total de parcelas a partir da fórmula proposta por Leite et al. (2009);
$T C H=(M 1 C \times N C M) \times 1000 / E S P$.Onde; M1C, refere-se a massa de média de um colmo, obtido por meio da pesagem, em $\mathrm{kg}$, de uma amostra aleatória de dez colmos (despontados); NCM é o número de colmos por metro linear, obtido pela razão entre contagem do número de colmos na parcela e os metros lineares da parcela; e ESP, refere-se ao espaçamento entre linha $(1,4 \mathrm{~m})$.

Para análise das famílias na fase T1, utilizouse do seguinte modelo estatístico associado à avaliação de famílias de irmãos-completos obtidas sob cruzamento dialélico desbalanceado, no delineamento de blocos incompletos, com uma observação por parcela, considerando o modelo descrito abaixo, conforme Resende (2006):

$$
y=X r+Z a+W f+U b+e
$$

Em que: $y, r, a, f, b, e$ : vetor de dados; dos efeitos de repetição (assumidos como fixos) somados à média geral; dos efeitos genéticos aditivos (assumidos como aleatórios); dos efeitos genéticos de dominância associado a famílias de irmãoscompletos (assumidos como aleatórios); dos efeitos dos blocos incompletos (aleatórios); e de erros ou resíduos (aleatórios), respectivamente. $X, Z, W$ e $U$ : representam as matrizes de incidência para os efeitos de $r, a, f$ e $b$, respectivamente.

Os parâmetros genéticos estimados foram: herdabilidade individual no sentido restrito $\left(h_{a}^{2}\right)$, herdabilidade individual no sentido amplo $\left(h_{g}^{2}\right)$, herdabilidade da média da familia no sentido amplo $\left(h_{m f}^{2}\right)$, variância genética aditiva $\left(\sigma_{a}^{2}\right)$, variância genética de dominância entre famílias $\left(\sigma_{d}^{2}\right)$, variância residual entre parcelas $\left(\sigma_{e}^{2}\right)$, variância fenotípica individual $\left(\sigma_{y}^{2}\right)$, coeficiente de determinação dos efeitos das famílias $\left(\mathrm{C}_{\text {fam }}^{2}\right)$, coeficiente de determinação dos efeitos do bloco $\left(\mathrm{C}_{\text {bloc }}^{2}\right)$, desvio padrão do valor genotípico predito (SEP), acurácia seletiva entre famílias em T1 $\left(\mathrm{AC}_{\mathrm{fam}}\right)$, coeficiente de variação genética $\left(\mathrm{CV}_{\mathrm{gi}} \%\right)$, coeficiente de variação ambiental $\left(\mathrm{CV}_{\mathrm{e}} \%\right)$, coeficiente de variação relativa $\left(\mathrm{CV}_{\mathrm{r}} \%\right)$ e Média Geral. 


\section{Fase T2}

A fase T2 foi constituída por clones selecionados das 22 melhores famílias, segundo a análise descrita anteriormente. No total, 195 clones foram selecionados na primeira fase de seleção (T1) do programa de melhoramento genético da cana-deaçúcar. O plantio da fase T2 foi realizado em 2003, no delineamento experimental de blocos aumentados (FEDERER, 1956). Cada bloco foi constituído por 18 clones e duas cultivares comerciais (padrões). A parcela experimental foi composta por duas linhas de cinco metros, espaçadas em 1,4 m entre-linhas, e com de plantio de 18 gemas por metro.

As avaliações realizadas foram; i) tonelada de cana por hectare $(\mathrm{TCH})$, conforme procedimento descrito anteriormente, e; ii) sanidade vegetal, com base na avaliação visual dos sintomas das principais doenças da cultura (carvão, ferrugem marrom e alaranjada, escaldadura e estria vermelha). As avaliações foram conduzidas nos meses de abril e julho de 2004, para os clones de ciclo de maturação precoce e maturação tardio, respectivamente.

Para análise dos clones dentro das famílias na fase T2, utilizou-se do seguinte modelo estatístico para a avaliação de clones no delineamento de blocos aumentados com uma observação por parcela:

$$
y=X f+Z g+W b+e
$$

Em que: $y, f, g, b, e$ : vetores de dados; de efeito fixo (média geral); de efeitos genotípicos de clones (aleatório); de efeitos de blocos (aleatório) e de erros aleatórios; respectivamente. $X, Z$ e $W$ : matrizes de incidência para $f, g$ e $b$, respectivamente.

Os parâmetros genéticos estimados neste modelo foram: herdabilidade individual no sentido amplo $\left(h_{g}^{2}\right)$, herdabilidade da média do genótipo, assumindo sobrevivência completa $\left(h_{m c}^{2}\right)$,variância genotípica $\left(\sigma_{g}^{2}\right)$, variância entre blocos $\left(\sigma_{b l}^{2}\right)$, variância residual entre parcelas $\left(\sigma_{\theta}^{2}\right)$, variância fenotípica individual $\left(\sigma_{y}^{2}\right)$, coeficiente de determinação dos efeitos do bloco $\left(\mathrm{C}_{\text {bloc }}^{2}\right)$, desvio padrão do valor genotípico predito (SEP), acurácia seletiva de genótipos em T2 $\left(\mathrm{AC}_{\text {clon }}\right)$, coeficiente de variação genética $\left(\mathrm{CV}_{\mathrm{gi}} \%\right)$, coeficiente de variação ambiental $\left(\mathrm{CV}_{\mathrm{e}} \%\right)$, coeficiente de variação relativa $\left(\mathrm{CV}_{\mathrm{r}} \%\right)$ e Média Geral.

O programa computacional utilizado para realizar as análises foi o Sistema Estatístico e Seleção Genética Computadorizada SELEGEN REML/BLUP, desenvolvido pela EMBRAPA (RESENDE, 2006).

A correlação entre BLUPIS x Seleção Massal foi calculada a partir do número de genótipos potenciais indicados pelo procedimento BLUPIS e o número de genótipos obtidos na seleção massal, para a fase $\mathrm{T} 2$ precoce $(\mathrm{P})$ e tardio $(\mathrm{T})$ e somatório de precoce e tardio. Do mesmo modo, procedeuse com a análise de correlação entre as médias genotípicas das famílias em T1 e com as médias genotípicas das famílias em T2, para o caractere TCH. A significância da correlação foi determinada pelo teste $t$ de Student.

Por fim, foi realizada uma análise comparativa entre as famílias que compuseram as fases T1, T2 e T3 do programa de melhoramento. Nesta análise, quantificou-se o número de clones selecionados dentro das famílias, sendo posteriormente estimado a porcentagem de seleção dentro destas. Foi realizado o teste de hipótese de Qui-quadrado $\left(\chi^{2}\right)$ de Pearson a fim de avaliar se as proporções observadas dentro das famílias mostraram ou não diferenças significativas.

\section{Resultados e Discussão}

Os resultados referentes às estimativas dos parâmetros genéticos para o caractere tonelada de cana por hectare $(\mathrm{TCH})$, avaliado nas famílias de irmãos-completos das fases $\mathrm{T} 1$ e $\mathrm{T} 2$ precoce $(\mathrm{P})$ e tardio (T), são apresentados na tabela 1. 
Tabela 1. Estimativa dos componentes de variância e parâmetros genéticos ${ }^{1}$ para tonelada de cana por hectare (TCH), em T1 e T2 precoce (P) e tardio (T). Curitiba, PR, 2012.

\begin{tabular}{lccc}
\hline Parâmetros & $\mathbf{T 1}$ & $\mathbf{T 2} \mathbf{( P )}$ & $\mathbf{T 2} \mathbf{( T )}$ \\
\hline$\sigma_{a}^{2}$ & 103,480 & - & - \\
$\sigma_{g}^{2}$ & - & 477,286 & 1226,480 \\
$\sigma_{b l}^{2}$ & - & 6,342 & 0,026 \\
$\sigma_{a}^{2}$ & 90,697 & 156,199 & 628,586 \\
$\sigma_{d}^{2}$ & 30,365 & - & - \\
$\sigma_{y}^{2}$ & 288,873 & 639,827 & 1855,092 \\
$h_{a}^{2}$ & $0,358+-0,176$ & - & - \\
$h_{g}^{2}$ & 0,779 & $0,746+-0,233$ & $0,661+-0,120$ \\
$h_{m f}^{2}$ & 0,945 & - & - \\
$h_{\text {me }}^{2}$ & - & 0,753 & 0,661 \\
$\mathrm{C}_{\text {bloc }}^{2}$ & 0,223 & 0,010 & 0,000 \\
$\mathrm{C}_{\text {fam }}^{2}$ & 0,105 & - & - \\
$\mathrm{AC}_{\text {fam }} ; \mathrm{AC}_{\text {clon }}$ & 0,972 & 0,868 & 0,813 \\
$\mathrm{CV}_{\text {gi } \%}$ & 18,814 & 21,933 & 31,941 \\
$\mathrm{CV}_{\text {}_{\%}}$ & 20,140 & 12,547 & 22,867 \\
$\mathrm{CV}_{r} \%$ & 0,934 & 1,748 & 1,397 \\
Média geral & 48,162 & 99,606 & 109,643 \\
\hline
\end{tabular}

${ }^{1}$ Herdabilidade individual no sentido restrito $\left(h_{a}^{2}\right)$, herdabilidade individual no sentido amplo $\left(h_{g}^{2}\right)$, herdabilidade da média da familia no sentido amplo $\left(h_{m f}^{2}\right)$, herdabilidade da média do genótipo, assumindo sobrevivência completa $\left(h_{m c}^{2}\right)$, variância genética aditiva $\left(\sigma_{a}^{2}\right)$, variância genotípica $\left(\sigma_{g}^{2}\right)$, variância genética de dominância entre famílias $\left(\sigma_{d}^{2}\right)$, variância entre blocos $\left(\sigma_{b l}^{2}\right)$, variância residual entre parcelas $\left(\sigma_{\theta}^{2}\right)$, variância fenotípica individual $\left(\sigma_{y}^{2}\right)$, coeficiente de determinação dos efeitos das famílias $\left(C^{2}{ }_{\text {fam }}\right)$, coeficiente de determinação dos efeitos do bloco $\left(\mathrm{C}^{2}{ }_{\text {bloc }}\right)$, desvio padrão do valor genotípico predito (SEP), acurácia seletiva entre famílias em T1/acurácia da seleção de genótipos em T2 $\left(\mathrm{AC}_{\mathrm{fam}} ; \mathrm{AC}_{\mathrm{clon}}\right)$, coeficiente de variação genética $\left(\mathrm{CV}_{\mathrm{gi}} \%\right)$, coeficiente de variação ambiental $\left(\mathrm{CV}_{\mathrm{e}} \%\right)$, coeficiente de variação relativa $\left(\mathrm{CV}_{\mathrm{r}} \%\right)$ e Média Geral.

Fonte: Elaboração dos autores.

Os coeficientes de variação genética $(\mathrm{CVg} \%)$ para a variável TCH indicaram haver variabilidade genética com possibilidade de seleção para aumento de produtividade, em todas as fases de seleção (T1, T2 P e T2 T), com valores de 19,57, 21,93 e $31,94 \%$, respectivamente (TAB. 1). Valores acima de $10 \%$, já indicam haver variabilidade genética com possibilidade de seleção, conforme relatado por Oliveira et al. (2011). Do mesmo modo, as estimativas de herdabilidade $\left(h_{a}^{2}\right.$ e $\left.h_{g}^{2}\right)$ evidenciaram bom controle genético na expressão do caractere $\mathrm{TCH}$, o qual indica grande potencial para seleção
(RESENDE, 2006). A herdabilidade em sentido amplo é um parâmetro importante em culturas de propagação vegetativa, como a cana-de-açúcar. Nestas espécies, a variabilidade genética é liberada uma única vez, e o genótipo de uma planta é fixado após o cruzamento, não havendo oportunidade de segregação nas fases futuras (ZHOU; JOSHI, 2012).

Outro parâmetro a ser considerado refere-se à acurácia da seleção dos genótipos, de forma que quanto maior o valor desse parâmetro na avaliação para um determinado caráter, maior é a confiança na avaliação e nos valores genotípicos preditos dos 
genótipos para um determinado caráter (RESENDE; DUARTE, 2007). Ao trabalhar com seleção de famílias, é possível elevar a acurácia seletiva de seleção de baixa para moderada a alta (RESENDE, 2006). A acurácia para as fases T1, T2 P e T2 T foram classificadas de magnitude alta a muita alta (RESENDE; DUARTE, 2007), com valores de $0,97,0,86$ e 0,81 , respectivamente (Tabela 1 ).

Os valores genotípicos (Vg) das 22 famílias selecionadas estão apresentados na tabela 2. Ao analisar a fase T1, das 22 famílias selecionadas 11 encontram-se acima da média destas melhores famílias $\left(50,96\right.$ t.ha $\left.^{-1}\right)$. Na seleção em T2 P, seis famílias superam a média geral das 22 famílias de 96,52 t.ha-1 e em T2 T, 11 famílias encontram-se acima da média de 111,96 t.ha ${ }^{-1}$ (Tabela 2). Neste sentido, para a melhor família (SP80-3280 X RB855589), o Vg em T1 de 63,85, aumenta para 97,44 em T2. Portanto, ao selecionar indivíduos nas famílias com elevado valor genotípico para a variável de interesse, como $\mathrm{TCH}$, a probabilidade de obter clones com Vg elevado é alta.

$\mathrm{Na}$ seleção via BLUPIS, famílias com efeitos genotípicos negativos são eliminadas automaticamente, por possuírem valor abaixo da média geral do experimento. Assim, haveria baixíssima probabilidade de se obter um clone promissor dentro destas famílias (RESENDE; BARBOSA, 2005; 2006).

A seleção das 11 melhores famílias em T1 proporcionou ganho médio de $12,21 \%$, considerando as mesmas famílias conduzidas na fase T2. Nesta fase, o ganho foi de $15,77 \%$, o que possibilitou ganhos acima da média em $50 \%$ das famílias que compuseram a fase $\mathrm{T} 2$.

$\mathrm{O}$ número de indivíduos selecionados em T1 por meio da metodologia BLUPIS e número de clones selecionados através de seleção massal e que compuseram a fase T2 (P e T) estão apresentados na tabela 3 .
A metodologia BLUPIS indicou a seleção de 226 indivíduos pertencentes a 11 famílias com efeitos genéticos positivos e que avançaram para a fase de seleção T2. Ao considerar as cinco melhores famílias, resultantes dos cruzamentos SP80-3280 x RB855589; SP80-3280 x RB835486; RB835486 x RB72454; SP80-1816 x RB855156; e RB835486 x SP83-2847, o número de indivíduos indicados foi 147 , o que representa aproximadamente $65 \%$ do total de indivíduos indicado pelo BLUPIS.

Na seleção massal, o número total de indivíduos selecionados dentro das 22 famílias foi de 182 no total, sendo 41 em T2 P e de 141 para T2 T (Tabela 3). Ao considerar somente as famílias de efeitos genotípicos positivos, o valor é reduzido para 99 indivíduos. Este valor é bem abaixo dos 226 indivíduos indicados via seleção BLUPIS. Deve-se lembrar que a seleção massal baseia-se em caracteres indiretos de produção, podendo levar a menor eficiência seletiva de indivíduos promissores para esta característica, pois apenas considera os efeitos fenotípicos (OLIVEIRA et al., 2011).

Por outro lado, a seleção massal proporciona eliminação antecipada de genótipos que apresentam sintomas de doenças. Ao analisar a seleção da melhor família via BLUPIS (SP803280 x RB855589), verifica-se a indicação de 39 indivíduos dentro da família, porém na seleção massal foram selecionados apenas dois indivíduos em T2 P e quatro indivíduos em T2 T. Uma explicação para este reduzido número de indivíduos selecionados dentro das melhores famílias seria decorrente da seleção massal ser realizada com base em características fenotípicas, sendo descartados indivíduos que possuíam sintomas de doenças ou algum caractere desfavorável, como por exemplo, pouco perfilhamento, entre outros. 
Tabela 2. Valores genotípicos (Vg) e média geral das famílias presentes em T1, T2 precoce (P) e T2 tardio (T) para a variável tonelada de cana por hectare (TCH). Curitiba, PR, 2012.

\begin{tabular}{|c|c|c|c|c|c|c|}
\hline \multirow{2}{*}{ Famílias } & \multicolumn{2}{|c|}{ T1 } & \multicolumn{2}{|c|}{ T2 (P) } & \multicolumn{2}{|c|}{$\mathrm{T} 2(\mathrm{~T})$} \\
\hline & $\mathrm{C}^{1}$ & $\mathbf{V g}$ & $\mathbf{C}$ & $\mathbf{V g}$ & $\mathbf{C}$ & Vg \\
\hline SP80-3280 x RB855589 & 1 & 63,85 & 5 & 97,44 & 18 & 96,10 \\
\hline SP80-3280 x RB835486 & 2 & 61,36 & 3 & 102,28 & 6 & 117,41 \\
\hline RB835486 x RB72454 & 3 & 60,31 & - & - & 11 & 112,73 \\
\hline SP80-1816 x RB855156 & 4 & 59,72 & 7 & 96,25 & 5 & 118,40 \\
\hline RB835486 x SP83-2847 & 5 & 56,98 & 11 & 66,28 & 12 & 111,15 \\
\hline RB855156 x SP70-1143 & 6 & 56,83 & 1 & 133,88 & 15 & 105,32 \\
\hline SP70-1143 x RB72454 & 7 & 56,53 & - & - & 4 & 122,87 \\
\hline IAC87-3396 x RB72454 & 8 & 55,30 & - & - & 1 & 172,36 \\
\hline RB72454 x L60-14 & 9 & 53,66 & - & - & 20 & 94,34 \\
\hline RB855186 x RB865547 & 10 & 52,59 & - & - & 19 & 95,19 \\
\hline RB72454 x IAC87-3396 & 11 & 51,86 & - & - & 9 & 113,06 \\
\hline RB855113 x RB835054 & 12 & 48,43 & - & - & 7 & 116,89 \\
\hline RB745464 x RB855127 & 13 & 48,41 & - & - & 2 & 154,35 \\
\hline TUC71-7 x RB72454 & 14 & 47,93 & 10 & 80,60 & 21 & 92,14 \\
\hline RB835054 x SP80-3280 & 15 & 47,84 & 6 & 96,51 & 3 & 125,48 \\
\hline RB72454 x CP70-1133 & 16 & 45,81 & 4 & 99,17 & 16 & 100,24 \\
\hline SP80-3280 x RB835054 & 17 & 45,34 & - & - & 10 & 112,98 \\
\hline RB835054 x SP83-2847 & 18 & 42,90 & 2 & 108,26 & 8 & 113,18 \\
\hline NA56-79 x SP80-3280 & 19 & 41,88 & 8 & 91,09 & 14 & 107,03 \\
\hline RB865547 x RB855186 & 20 & 41,74 & 9 & 89,96 & 17 & 98,06 \\
\hline NA56-79 x RB72454 & 21 & 40,90 & - & - & 22 & 74,46 \\
\hline RB72454 x NA56-79 & 22 & 40,90 & - & - & 13 & 109,34 \\
\hline Média das 22 famílias selecionadas & & 50,96 & & 96,52 & & 111,95 \\
\hline Média das 40 famílias & & 49,67 & & 99,60 & & 109,64 \\
\hline
\end{tabular}

${ }^{1} \mathrm{C}$ - classificação em relação ao $\mathrm{Vg}$.

Fonte: Elaboração dos autores. 
Tabela 3. Número de indivíduos selecionados dentro das famílias em T1 via método BLUPIS (variável TCH) e número de clones selecionados em T2 precoce $(\mathrm{P})$ e tardio $(\mathrm{T})$ via seleção massal em famílias de irmãos- completos de cana-de-açúcar. Curitiba, PR, 2012.

\begin{tabular}{|c|c|c|c|c|}
\hline \multirow{2}{*}{$\mathbf{C}^{1}$} & \multicolumn{2}{|l|}{ T1 } & \multirow{2}{*}{$\begin{array}{c}\text { T2 (P) } \\
\text { Clones sel. }\end{array}$} & \multirow{2}{*}{$\begin{array}{c}\text { T2 (T) } \\
\text { Clones sel." }\end{array}$} \\
\hline & Família & Blupis $\left(n_{k}\right)\left(n_{j}=50\right)$ & & \\
\hline 1 & SP80-3280 x RB855589 & 39 & 4 & 2 \\
\hline 2 & SP80-3280 x RB835486 & 32 & 11 & 21 \\
\hline 3 & RB835486 x RB72454 & 29 & ** & 13 \\
\hline 4 & SP80-1816 x RB855156 & 27 & 13 & 8 \\
\hline 5 & RB835486 x SP83-2847 & 20 & 1 & 4 \\
\hline 6 & RB855156 x SP70-1143 & 20 & 1 & 2 \\
\hline 7 & SP70-1143 x RB72454 & 19 & ** & 11 \\
\hline 8 & IAC87-3396 x RB72454 & 15 & ** & 1 \\
\hline 9 & RB72454 x L60-14 & 11 & ** & 3 \\
\hline 10 & RB855186 x RB865547 & 8 & ** & 2 \\
\hline 11 & RB72454 x IAC87-3396 & 6 & ** & 2 \\
\hline 12 & RB855113 x RB835054 & 0 & ** & 5 \\
\hline 13 & RB745464 x RB855127 & 0 & ** & 15 \\
\hline 14 & TUC71-7 x RB72454 & 0 & 2 & $* *$ \\
\hline 15 & RB835054 x SP80-3280 & 0 & 4 & 11 \\
\hline 16 & RB72454 x CP70-1133 & 0 & 2 & 5 \\
\hline 17 & SP80-3280 x RB835054 & 0 & ** & 22 \\
\hline 18 & RB835054 x SP83-2847 & 0 & 1 & 5 \\
\hline 19 & NA56-79 x SP80-3280 & 0 & 1 & 2 \\
\hline 20 & RB865547 x RB855186 & 0 & 1 & 4 \\
\hline 21 & NA56-79 x RB72454 & 0 & ** & 2 \\
\hline 22 & RB72454 x NA56-79 & 0 & ** & 1 \\
\hline & Total & 226 & 41 & 141 \\
\hline
\end{tabular}

${ }^{1} \mathrm{C}$ : classificação de famílias com base no número de genótipos selecionados pelo procedimento BLUPIS; *: Numero de clones selecionados dentro da família. **: Indivíduos não selecionados dentro das famílias.

Fonte: Elaboração dos autores.

Ao comparar os procedimentos de seleção via BLUPIS em T1 com a seleção massal (número de clones obtidos para o T2) verificou-se correlação significativa de $\mathrm{r}=0,72^{* *}$ (Tabela 4). Este resultado evidencia a eficiência da seleção via BLUPIS, e informa que $72 \%$ dos clones selecionados em T2 são procedentes das famílias com efeitos genotípicos positivos. Famílias estas que foram avaliadas na fase T1 e indicadas pelo procedimento BLUPIS. Este resultado corrobora com o verificado por Oliveira et al. (2011), o qual encontraram $r=0,66^{* *}$ entre a seleção via BLUPIS e a seleção massal. 
Tabela 4. Estimativa dos coeficientes de correlação de Pearson entre T1 e T2 tardio (T), T1 e T2 precoce (P), T1 e T2 a partir de 40 famílias de irmãos- completos da série RB01. Curitiba, PR, 2012.

\begin{tabular}{lcc}
\hline \multicolumn{1}{c}{ Descrição } & $\mathbf{r}$ & $\boldsymbol{\alpha}$ \\
\hline Blupis T1 e clones em T2 (T) & $0,43^{\text {ns }}$ & 0,060 \\
Media TCH T1 e T2 (T) & $-0,16^{\text {ns }}$ & 0,496 \\
Blupis T1 e clones em T2 (P) & $0,76^{*}$ & 0,019 \\
Media TCH T1 e T2 (P) & $0,18^{\text {ns }}$ & 0,659 \\
Blupis em T1 e clones em $\sum$ T2 & $0,72^{* *}$ & 0,008 \\
Média TCH T1 e T2 & $0,07^{\text {ns }}$ & 0,844 \\
\hline
\end{tabular}

ns: não significativo; * significativo a $5 \%$ de probabilidade; ** significativo a $1 \%$ de probabilidade.

Fonte: Elaboração dos autores.

Esta correlação significativa comprova que as duas metodologias indicam resultados semelhantes, porém na seleção massal há tendência de selecionar maior número de indivíduos oriundos de cruzamentos abaixo da média experimental, como foi obtido para o $\mathrm{TCH}$.

Por outro lado, não se verificou correlação entre as médias de produtividades das famílias em T1 e T2 $(r=0,07)$. Os baixos valores de correlação também são observados entre as médias de TCH entre BLUPIS em T1 e T2 P $(-0,16)$ e T2 T $(0,18)$. Isto se deve à seleção de $\mathrm{TCH}$ envolver caracteres indiretos, que podem ser influenciados nos diferentes ambientes e fases de seleção.

A porcentagem de seleção dentro das famílias, na fase $\mathrm{T} 1$ variou de $0,5 \%$ a $16,9 \%$ e na fase $\mathrm{T} 2$ variou de 3,3\% a 8,3\% (Figura 1). Das 40 famílias avaliadas em T1 foram selecionadas 22 para compor T2, com base nos caracteres indiretos de seleção para $\mathrm{TCH}$, sendo estes: massa de um colmo (M1C kg), número de colmos por touceira (NCT), número de colmos por metro linear (NCM) e massa de uma touceira (M1T kg). As sete famílias que apresentaram maior porcentagem de seleção foram resultado do cruzamento entre os genitores: RB835054 x SP83-2847; RB72454 x CP70-1133; RB835486 x SP83-2847; RB835054 x SP80-3280; SP80-1816 x RB855156; SP80-3280 x RB855589; e SP80-3280 x RB835486; e suas respectivas porcentagens de seleção foram de 6,$2 ; 7,2 ; 9,7 ; 12,3 ; 12,8$ e 16,9\%, respectivamente.
As melhores famílias selecionadas na fase $\mathrm{T} 2$ e que constituíram a fase T3 foram sete, resultado dos cruzamentos entre: RB835486 x RB72454; RB855156 x SP70-1143; RB835054 x SP83-2847; RB835054 x SP80-3280; SP80-1816 x RB855156; SP80-3280 x RB855589; e SP80-3280 x RB835486. A porcentagem de seleção destas famílias foi de: 8,3 ; 8,$3 ; 8,3 ; 8,3 ; 16,6 ; 33,3$ e $16,6 \%$ respectivamente sendo cinco em comum com as que apresentaram maiores porcentagens na seleção via procedimento BLUPIS em T1 (Figura 1).

A maior porcentagem de clones selecionados em T1 (16,9\%) foi obtida pela família oriunda do cruzamento SP80-3280 x RB835486 (Figura 1). Na sequência, aparece com o segundo maior valor de seleção na fase T2 (16,7\%). Pelo método BLUPIS, esta família alcançou a segunda posição (Tabela 3) no número de clones a serem selecionados em T1 (32 indivíduos indicados pelo BLUPIS). Este mesmo resultado se repete quando observada a seleção massal em T2, onde esta mesma família está presente com elevados valores de seleção, sendo 11 indivíduos selecionados em T2 P e 21 indivíduos selecionados em T2 T (segundos maiores valores na seleção massal).

Verificou-se que algumas famílias, com altas porcentagens de seleção na fase $\mathrm{T} 2$, apresentaram seleção nula ou baixa na fase T3. Como exemplo, tem-se as famílias resultantes dos cruzamentos; RB72454 x NA56-79, SP80-3280 x RB835054, RB72454 x CP70-1133 e RB835486 x SP83-2847. 
Estas famílias apresentaram sintomas de carvão, o que resultou na eliminação completa da família na fase T3. O mesmo aconteceu com a família resultante do cruzamento SP80-3280 x RB835486, que poderia ter tido maiores taxas de seleção em T3. Entretanto, esta família apresentou incidência de ferrugem alaranjada, sendo descartados os clones que apresentavam sintomas.

Figura 1. Porcentagem de clones selecionados dentro das famílias nas fases do melhoramento. A) Clones selecionados em T1 para constituir a fase T2. B) Clones selecionados na fase T2 para constituir a fase T3. Curitiba, PR, 2012.
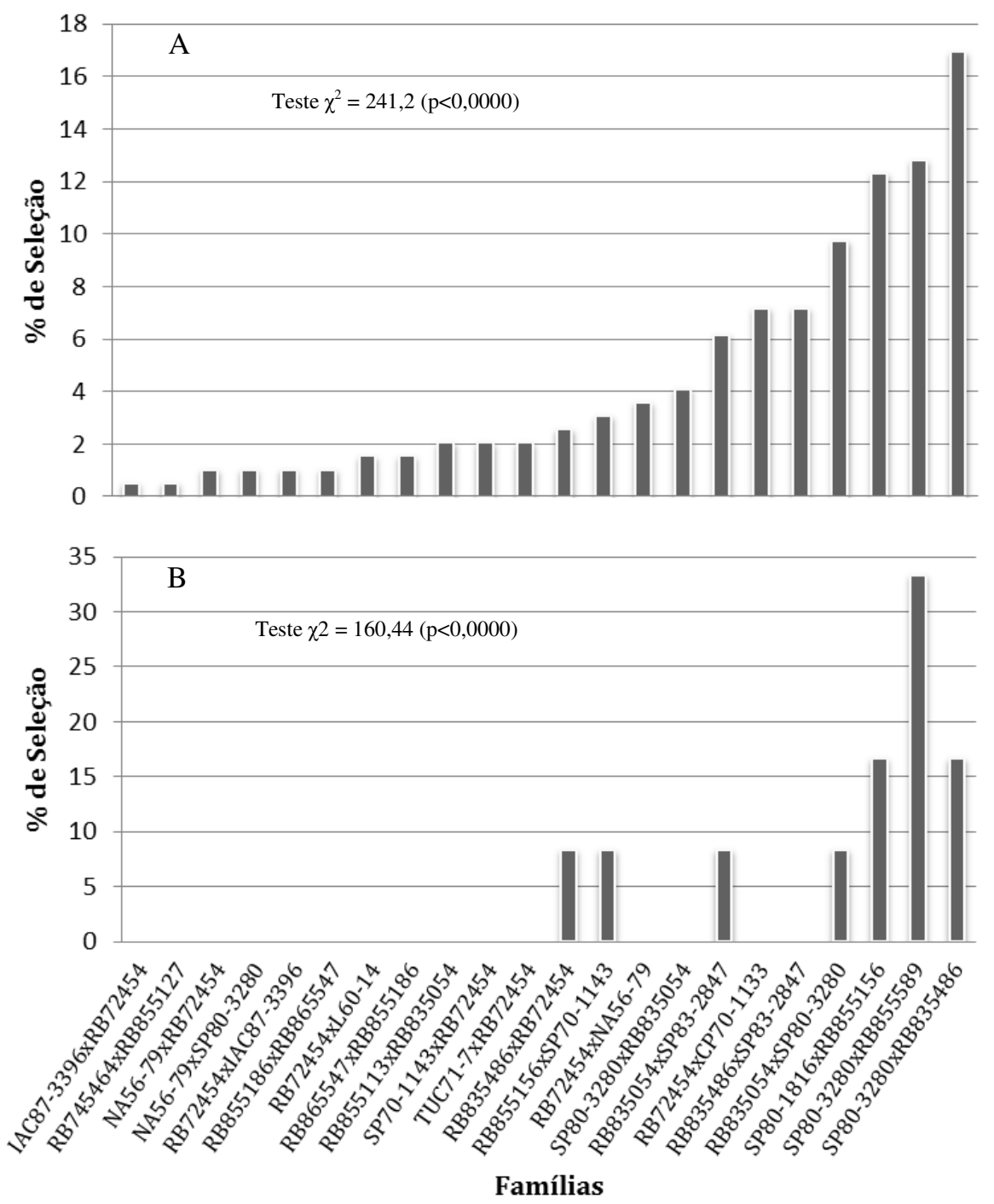

Fonte: Elaboração dos autores.

\section{Conclusões}

A seleção utilizando informações geradas pelo método BLUPIS, dentro das famílias com valores genotípicos acima da média experimental na fase T1, pode possibilitar maior taxa de clones selecionados na fase T2 e com maior probabilidade de avançar para as etapas seguintes do melhoramento genético;

As melhores famílias na fase $\mathrm{T} 1$, e que foram testadas nas fases seguintes, possibilitaram a obtenção de maior número de clones promissores 
e neste estudo foram provenientes dos cruzamentos entre: SP80-1816 x RB855156; SP80-3280 x RB855589 e SP80-3280 x RB835486.

\section{Referências}

ARNHOLD, E.; VIANA, J. M. S.; SILVA, R. G. Associação de desempenho entre famílias S3 e seus híbridos top cross de milho-pipoca. Revista Ciência Agronômica, Fortaleza, v. 40, n. 3, p. 396-399, 2009.

BARBOSA, M. H. P.; RESENDE, M. D. V.; BRESSIANI, J. A.; SILVEIRA, L. C. I.; PETERNELLI, L. A. Selection of sugarcane families and parents by REML/BLUP. Crop Breeding and Applied Biotechnology, Viçosa, v. 5, n. 4, p. 443-450, 2005.

BARBOSA, M. H. P.; RESENDE, M. D. V.; PETERNELLI, L. A.; BRESSIANI, J. A.; SILVEIRA, L. C. I.; SILVA, F. L.; FIGUEIREDO, I. C. R. Use of REML/ BLUP for the selection of sugarcane families specialized in biomass production. Crop Breeding and Applied Biotecnology, Viçosa, v. 4, n. 2, p. 218-226, 2004.

BARRETO, R. R.; SCAPIM, C. A.; AMARAL JÚNIOR, A. T.; RODOVALHO, M. A.; VIEIRA, R. A.; SCHUELTER, A. R. Avaliação da capacidade de combinação de famílias S2 de milho-pipoca por meio de diferentes testadores. Semina: Ciências Agrárias, Londrina, v. 33, n. 3, p. 873-890, 2012.

DUTRA FILHO, J. A.; MELO, L. J. O. T.; RESENDE, L. V.; ANUNCIAÇÃO FILHO, C. J.; BASTOS, G. Q. Aplicação de técnicas multivariadas no estudo da divergência genética em cana-de-açúcar. Revista Ciência Agronômica, Fortaleza, v. 42, n. 1, p. 185-192, 2011.

FEDERER, W. T. Augmented (or Hoonuiaku) designs. Hawaiian Planters' Records, Aiea, v. 55, n. 2, p. 191208, 1956.

GIORDANI, S. C. O.; FERNANDES, J. S. C.; TITON, M.; SANTANA, R. C. Parâmetros genéticos para caracteres de crescimento em pequizeiro em estádio precoce. Revista Ciência Agronômica, Fortaleza, v. 43, n. 1, p. 146-153, 2012.

KIMBENG, C. A.; COX, M. C. Early generation selection of sugarcane families and clones in Australia: a review. Journal American Society of Sugarcane Technologists, Baton Rouge, v. 23, n. 6, p. 20-39, 2003.

LEITE, M. S. O.; PETERNELLI, L. A.; BARBOSA, M. H. P.; CECON, P. R.; CRUZ, C. D. Sample size for full-sib family evaluation in sugarcane. Pesquisa Agropecuária Brasileira, Brasília, v. 44, n. 12, p. 15621574, 2009.
OLIVEIRA, R. A.; DAROS, E.; BESPALHOK-FILHO, J. C.; ZAMBON, J. L. C.; IDO, O. T.; WEBER, H.. RESENDE, M. V. D.; ZENI-NETO, H. Seleção de famílias de cana-de-açúcar via modelos mistos. Scientia Agrária, Curitiba, v. 9, n. 3, p. 269-274, 2008.

OliVEIRA, R. A.; DAROS, E.; RESENDE, M. D. V.; BESPALHOK-FILHO, J. C.; ZAMBON, J. L. C.; DE SOUZA, T. R.; FERNANDEZ LUCIUS, A. S. Procedimento Blupis e seleção massal em cana-deaçúcar. Bragantia, Campinas, v. 70, n. 4, p. 1-5, 2011.

PEDROZO, C. A.; BARBOSA, M. H. P.; VILELA, M. D.; PETERNELLI, R. L. A.; COSTA, P. M. A.; SILVA, F. L. Eficiência de seleção em fases iniciais do melhoramento da cana-de-açúcar. Revista Ceres, Viçosa, v. 55, n. 1, p. 1-8, 2008.

RESENDE, M.D. V.; BARBOSA, M. H. P. Melhoramento genético de plantas de propagação assexuada. Colombo: Embrapa Informação Tecnológica, 2005. 130 p.

RESENDE, M. D. V.; BARBOSA, M. H. P. Selection via simulated BLUP based on family genotypic effects in sugarcane. Pesquisa Agropecuária Brasileira, Brasília, v. 41, n. 3, p. 421-429, 2006.

RESENDE, M. D. V.; DUARTE, J. B. Precisão e controle experimental de qualidade em experimentos de avaliação de cultivares. Pesquisa Agropecuária Tropical, Goiânia, v. 37, n. 3, p. 182-194, 2007.

RESENDE, M. D. V. SELEGEN - REML/BLUP. Sistema estatístico e seleção genética computadorizada via modelos lineares mistos. Colombo, PR: Embrapa Florestas, 2006. 359 p.

ROCHA, M. D. B.; PIRES, I. E.; XAVIER, A.; CRUZ, C. D.; ROCHA, R. B. Avaliação genética de progênies de meio-irmãos de Eucalyptus urophylla utilizando os procedimentos REML/BLUP e E(QM). Ciência Florestal, Santa Maria, v. 16, n. 4, p. 369-379, 2006.

SILVA, G. O.; VIEIRA, J. V.; NASCIMENTO, W. M. Estratégias de seleção para germinação de sementes de cenoura em altas temperaturas. Semina: Ciências Agrárias, Londrina, v. 32, n. 3, p. 849-854, 2011.

STRINGER, J. K.; COX, M. C.; ATKIN, F. C.; WEI, X.; HOGARTH, D. M. Family selection improves the efficiency and effectiveness of selecting original seedlings and parents. Sugar Tech, Kunraghat, v. 13, n. 1, p. 36-41, 2011.

ZHOU, M.; JOSHI, S. Trends in broad sense heritability and implications for sugarcane breeding in South Africa. Sugar Tech, Kunraghat, v. 14, n. 1, p. 40-46, 2012. 
